\title{
An Exploration on the Developing of Expert System in Transport Engineering
}

\author{
Shuhairy Norhisham ${ }^{1, *}$, Amiruddin Ismail $^{2}$, Muhamad Nazri Borhan ${ }^{2}$, and Herda Yati \\ Katman $^{1}$ \\ ${ }^{1}$ Department of Civil Engineering, College of Engineering, Universiti Tenaga Nasional \\ ${ }^{2}$ Department of Civil and Structural Engineering, Faculty Engineering and Built Environment, \\ Universiti Kebangsaan Malaysia
}

\begin{abstract}
This paper aims to determine the expert system developed from past research observations. This overview of expert system indicates the use of system which have slight affects on the results. Planning of research design enables a researcher to decide on what system it should be conducted. Two main components categorized for this paper are knowledge base obtained from previous study and a comparative study between the expert system to get a summary of the best inference engine, knowledge base, benefit and understanding.
\end{abstract}

\section{Introduction}

The expert system is subjected to smart computers that basically solve any intriguing difficulty and the transport engineering area is matured for development. Generally, an expert system is about incorporating human expertise into computer program to solve any problems requiring a human expert [14]. In order to use the expert system, planning and operational should be firstly identified. The purpose for planning is to determine the margins of share for specific transport modes while the operational purpose is to provide the survey with parameter values from the existing state or for making a short-term forecast for these parameters [12]

To identify the characteristics for the expert system, [15] conducted a survey on the classification by function in four very broad groups. They were traffic management control; traffic impact and safety; highway design and planning; and highway management. All these boards were surveyed according to their functions.

Consequently, the ability of expert system to access regular updated regulations from commercial programs was one requirement for the expert system [8].

In other hand, developing expert system can assist and advice engineers in dealing with traffic safety problem. Hence, the gap between traffic safety and people (decision makers, engineers and students) would be filled [4].

\footnotetext{
*Corresponding author: shuhairy@uniten.edu.my
} 


\section{Expert system evaluation proses}

Developing an expert system should combine with the evaluation process. The evaluation process employed for expert systems differs substantially from that used for traditional software projects and is much more problematic, mainly because of the nature of problems being solved by the expert system [14].

Verification, validation and evaluation are three processes that should be measured. Verification is the consistency, completeness and correctness of the software. Hence for software verification it totally determines if the system was built according to specifications. While validation is concerned with the quality of the conclusions. Thus it focuses on the effectiveness of system. A commonly borrowed phraseology to describe the difference between verification and validation is that verification is concerned with building the system right, whereas validation is concerned with building the right system.

Meanwhile evaluation process is concerned with user issues such as user acceptance and system usefulness. Therefore, with respect to all of above, validation is the cornerstone of system evaluation process and is the most difficult component to accomplish.

\section{Expert system definition}

Expert system is a computer system that emulates or acts in all respects, with a human expert decision making capabilities. Generally, the system contains knowledge base of accumulated experience and a set of rules for applying the knowledge base to each particular situation that is described to the program.

[12] defined the expert system as the creation of basic idea on the modal spilt against basis parameter for transport system demand and supply in the city. Meanwhile [14] described the expert system as a computer program that react to solve problems and require a human expert. Besides that, an expert system can solve problem requiring judgement and experience if the problem is complex or have inadequate information input.

On the other hand, [10] named the expert system as an advisory system which contains advice for user and assist problem solving that are normally solved by human expert. According to [4], expert system is also known as computer based programs that are developed to mimic and imitate problem solving processes along with the reasoning of human expert in different knowledge fields. Thus, this expert system slightly assist human in solving problem which require extensive knowledge or a long time.

\subsection{Processes of building expert system}

Expert system is currently used as support methods that are developed for best result. This paper is currently searching for process of building expert system knowledge, they include:

i) The knowledge engineer establishes a dialog with the human expert to elicit knowledge.

ii) The knowledge engineer codes the knowledge explicitly in the knowledge base.

iii) The expert evaluates the expert system and gives a critique to the knowledge engineer. 


\section{Application of expert system in transport engineering}

\subsection{Automated fare}

The automated fare collection is introduced by using smart card technology and electronic payment system. [11] explored the essential to integrate effective information technology management in public transport strategic information technology planning. It involved how essential it is to integrate effective information technology management with the public transport strategic information technology. The reason why strategic information technology planning is needed is because the system is a core instrument for communication within organizations and to external stakeholders and customers. Basically, the function of automated fares is to ease the seamlessness of multi-modal passengers to travel. The automated fare collection could address many key issues especially with regards to fare collection faced by the public transport agency [11].

\subsubsection{Advantage}

With all the motivation and opportunities that drove the multi-modal automated fare collection solution, several rationales benefit from the system including [11]:

1. Reduce maintenance and operational costs

2. Increase transaction throughput for travelers

3. Reduce fraudulence activities. Innovations for ticketing and fare options

4. Flexible fare pricing

\subsection{Geographic information system for transport (GIS-T)}

[16] developed GIS as a computer-based system which could manage objects and their information with their spatial characteristics. Basically, GSI-T is a geographical information system for transport, created by combining the advantages of both GISs and TIS to store geographic information, visualize, plan, and analyze transport information. Then, [5] introduced GIS-T software as interconnected systems that consist of hardware, software, data, people organization and institutional arrangements for collecting, storing, analyzing and disseminating information about areas on earth that are used, influenced or affected by transport activity

The study aimed to evaluate bus delays based on some factors such as traffic congestion, passenger's waiting time and the bus line interaction [16].

In this study, the GIS-T provided a database of detailed information to support simulations. A digital map was coordinated to the system as a background map. The background map contained information on block, road, railway, water area and green area. Then, all the bus lines and bus stations were located on the digital map with the coordinates.

\subsubsection{Advantage}

The software is simple and has a convenient interface. It is also a direct representation of the network that is constructed on supported background pictures. Then, by providing the advanced signal control mechanism it makes simulations of crossroads 
intersection closer to reality. Other than that, the software usually customized results so that the user can control data collection speed by adjusting the sampling time. Besides that the software can seamless interface with the network constructed in VISUM would be imported into VISSIM to simulate. Meanwhile the simulated VISSIM network must be transferred into VISUM to get a detailed graphical representation like cobweb graphs. In addition, the urban traffic simulation accuracy is well improved because of the Innovative pedestrian simulation.

\subsection{System Dynamics (SD)}

System Dynamics (SD) is a methodology of wide applicability and pioneered the use of system concepts and computer simulation for complex problems analysis. This system has become a modelling style used by various disciplines.

This system provides a framework structure through a large easily accomodated scale system, Other than that, the socio-economic and demographic forecasts are obtained by using separate modelling techniques. Then, SD models also utilize easily available data which is mainly required to initialize the model for simulation run. Besides that, using SD for transport modelling, the way in which traditional submodel are linked to each other and to other subsystems, occur simultaneously rather than step by step. In addition, results of SD transport model are achieved through the dynamic, casual, feedback interactions of the model structural components.

\subsection{Case-Based Reasoning/ Rule Based Reasoning}

Case-Based Reasoning can be defined as the program that acts as solution to unsolved problems based on pre-existing solutions of a similar nature.

On the other hand, CBR is also known as brilliant techniques to solve human problem and new problems will be solved by recalling and adapting the solutions of similar past problems. CBR is also one of the famous and active research area since many companies have adopted the program.

Rule based reasoning is used to store and manipulate knowledge to interpret useful information. $\mathrm{RBC}$ is also known as simple patterns and an inference engine that searches for patterns in the rules that match patterns in the data.

- Function: CBR functions as Crew Scheduling Planning According, Crew Scheduling, Crew Attendance Management and Weekends Shift [9].

- Advantage: CBR and RBR give some benefits that include representing the rules neutrality that represent method with high comprehensibility level. Then, modularity reflects a rule with discrete knowledge unit that can be inserted into or removed from the knowledge base. Other than that, it provides compact representation of general knowledge [13].

\subsection{Radioactive Materials Prototype}

The Radioactive Materials Prototype is initially built to demonstrate the feasibility of building an expert system. The system is built according to module for transporting and packaging radioactive materials. Then the module is developed with respect to hazardous chemicals. 
In order to build the system, the feasibility stage included in few steps. Firstly, the commercial software that was related to regulation access was analyzed. Secondly, the knowledge acquisition was obtained. Finally, the expert system prototype was developed [8].

The main function for this system is to ensure straight forward, consistent and errorfree application towards regulations for hazardous materials transport [8].

\subsection{Integrated Transportation Systems}

Multilayer and Multilevel Space of Integration is a natural approach for transport system. ITS is an integration of a number of technologies consisting of information processing, communication, control and an electronic intelligent link between travelers, vehicles and infrastructure. This clearly shows that ITS offers problem solving for transport problems [1].

From the knowledge base (KB) aspect, the system application includes traffic management, supervision and control system that were presented as motivated by several important reasons. The traffic control problems were normally well-structured and formulated to be solved by purely algorithmic techniques [1].

ITS provide KB system with problem solving methods, mechanisms working with some degree of multiple tasking and problem domain tools for automatic generation and customization of dedicated knowledge [1].

\subsubsection{Advantage}

The system provides many benefits through the traditional system that result in provision of area-wide transport demand management. Other than that, it also gives area wide synchronized response to the full range of mobility needs. Development on the efficient multi-layered technical provide opportunity to multi modal planning [1].

\subsection{E-SUTRA}

E-SUTRA is an engineering software that is developed as a problem solving expert system. The expert system shell was developed for young and inexpert transport engineers and planners who are currently involved in transport demand management (TDM) strategy planning and implementation. As for the knowledge base model, the nine class module is made up of each deal with specific strategy [10].

This system functions as a decision support system that represent a teaching tool for transport engineers, planners, private developers and also government officials. The system has reliable advice that can efficiently decide on the Transport Demand Management strategic planning and implementation process. In other hand, this system provided knowledge, skills and experiences; because they can undertake a complex scenario analysis [10].

The expert system is user friendly and it does not require the user to have experience or knowledge on programming. This is because the user can smoothly navigate through nine different input screens before the system outputs its summary. On the other hand, the system help young and fresh engineers and planner in their decision making. Moreover, the system is very costly and time saving [10]. 


\subsection{Intelligent Electronic Devices (IEDs)}

[2] proposed IEC 61850-9-2 as a developer system that operates protection IEDs performance in terms of dependability, security, and operating speed. The system is an internationally recognized non-proprietary standard for power system substation communication networks and automation. The system currently detects the process bus with a reliability of power system protection. IEDs real protection was used in hardware in-the-loop set-up with the RTDS. It is important to use the actual process bus equipment when carrying out performance analysis to investigate and record the equipment performance [2].

The advantage of implementing this system is a reduction in the overall cost of substation protection, automation and control system because the cost of numerous copper conductor is reduced. Moreover, the system is safer to use and the IEC 618509-2 permits wide availability of measurements to individual protection IEDs on the digital communication bus and system reconfiguration can be done in less time with a minimum cost as compared to the conventional hardwired method. To summarize, the system benefits substations safety, utilize less copper, facilitate maintenance and easy reconfiguration, etc. while giving the same performance in terms of operational speed, security, and dependability available from conventional analogue inputs IEDs [2].

\subsection{CALMSYS}

CALMSYS is a web-based traffic calming expert system developed to assist engineers improve safety all road-users and residents in residential streets safety [4].

The system is divided into three sections namely Knowledge Acquisition, Selection of Building Tool and Knowledge Based. For Knowledge Acquisition, the expert system is obtained from human expertise in traffic calming and written sources of knowledge. Meanwhile for the Selection of Building tool, Microsoft Visual Basic.NET is widely used for developing expert system and the software is easy to learn with flexible language that enables coding and creates GUIs (graphical user inter- faces). For Knowledge Based, the system works independently but the collaboration between traffic calming strategies modules existed [4].

\subsubsection{Advantage}

The system is important because it classifies the strategies and solutions for traffic calming subject. The strategies are categorized in physical, nonphysical and combined categories. In addition, the system can assist engineers in finding proper traffic calming strategies for traffic safety problems. Moreover, by this system can help in better and more succeful handling with a computerized system rather than with human experts due to the presence of many data received [4].

\section{Discussion and conclusion}

In conclusion, the expert system has its own benefits: increased availability, reduced cost, reduced danger, performance, multiple expertise, increased reliability, explanation, fast response, steady, unemotional, and complete responses at all times, intelligent tutor and intelligent database. 
The reviewed expert system in this study express the importance of applying modern technology in research design. In analyzing transport engineering, the most appropriate system must be introduced during the research study. The system can affect the achieved results. Several systems may be used but not all the system are appropriate for the research study. Therefore, in conclusion computer based simulation can provide decision-makers with strategies for intermodal terminals development.

\section{References}

[1] A. Adamski, Intelligent integrated transportation systems, The 9th Meeting of the EURO WG Intermodality, Sustainability and ITS, Bari, 565-570 (2002)

[2] A.C. Adewole, R. Tzoneva, Impact of IEC 61850-9-2 standard-based process bus on the operating performance of protection IEDS : Comparative study, The 19th World Congress The International Federation of Automatic Control, Cape Town, South Africa, 2245-2252 (2014)

[3] P. Chandra, P. Soni, R.K. Keshari, RFID-based ticketing for public transport system : Perspective megacity, Int. J. of Advance Research in Computer Science and Management Studies, 7782, 187-191 (2014)

[4] A. Falamarzi, M.N. Borhan, R.A.O.K. Rahmat, Developing a web-based advisory expert system for implementing traffic calming strategies, The Scientific World J., (2014)

[5] D. R. Fletcher, Geographic Information Systems for Transportation: A Look Forward, Transportation in the New Millenium: State of the Art and Future Directions, (2000)

[6] V. Graudina, J. Grundspenkis, Technologies and multi-agent system architectures for transportation and logistics support: An overview, In International Conference on Computer Systems and Technologies, 1-6 (2005)

[7] E.J. Heikkila, T. Kim, J.M. II, Incorporating Expert Geographic Information Systems Into Urban Land Use and Transportation Planning Models, In Future Directions For EGIS: Application to Land Use and Transportation Planning, (1989)

[8] J.J. Ferrada, D. Micbelhaugh, R. Rawl, development of an expert system for transportation of hazardous and radioactive materials, International Nuclear and Hazardous Waste Management, (1994)

[9] T. Liu, J. Ma, W. Guan, Y. Song, P. Fu, Design and implementation of bus crew scheduling system using integrated case-based and rule-based reasoning, 2012 Fifth International Joint Conference on Computational Sciences and Optimization, 475-479 (2012)

[10] R. Mansyur, R.A.O.K. Rahmat, A. Ismail, M.R. Kabit, Decision suport system for transport demand mangement: Object oriented approach using Kappa Pc 2 . 4 expert system shell, ARPN J. of Engineering and Applied Sciences, 6(2), 73-81 (2012)

[11] N.M. Noor, A multimodal automated fare collection solution for facilitating strategic information technology planning of public transportation in Malaysia. International Symposium on Information Technology, 1-8, (2008)

[12] M.M. Popović, J.J. Jović, Concept of expert system for modal split in transportation planning, Yugoslav J. of Operations Research, 16(1), 107-124 (2006) 
[13] J. Prentzas, I. Hatzilygeroudis, Categorizing approaches combining rule-based and case-based reasoning, Expert Systems 24.2, 1-34 (2007)

[14] G.S. Spring, Critical review of expert system validation in transportation, J. of the Transportation Research Board, (970106), 8-12 (1997)

[15] J.A. Wentworth, Expert systems in transportation, 24-29 (1993)

[16] L. Zhang, X. Ren, A two-factor evaluation of bus delays based on GIS-T database and simulation, (2010)

[17] M. Zouari, C. Diop, E. Exposito, Multilevel and coordinated self-management in autonomic systems based on service bus, J. of Universal Computer Science, 20(3), $431-460(2014)$ 